\title{
Anisotropic invasion and its consequences in two-strategy evolutionary games on a square lattice
}

\author{
György Szabó \\ Institute of Technical Physics and Materials Science, Centre for Energy Research, Hungarian Academy of Sciences, \\ P.O. Box 49, H-1525 Budapest, Hungary \\ Levente Varga \\ Babeş-Bolyai University, Faculty of Physics, RO-400084 Cluj-Napoca, Romania \\ Mátyás Szabó \\ Reed College, Department of Physics, 97202 Portland, Oregon, USA
}

(Received 25 August 2016; revised manuscript received 26 October 2016; published 22 November 2016)

\begin{abstract}
We have studied invasion processes in two-strategy evolutionary games on a square lattice for imitation rule when the players interact with their nearest neighbors. Monte Carlo simulations are performed for systems where the pair interactions are composed of a unit strength coordination game when varying the strengths of the self-dependent and cross-dependent components at a fixed noise level. The visualization of strategy distributions has clearly indicated that circular homogeneous domains evolve into squares with an orientation dependent on the composition. This phenomenon is related to the anisotropy of invasion velocities along the interfaces separating the two homogeneous regions. The quantified invasion velocities indicate the existence of a parameter region in which the invasions are opposite for the horizontal (or vertical) and the tilted interfaces. In this parameter region faceted islands of both strategies shrink and the system evolves from a random initial state into the homogeneous state that first percolated.
\end{abstract}

DOI: 10.1103/PhysRevE.94.052314

\section{INTRODUCTION}

Evolutionary game theory was originally developed to describe the macroscopic behavior of multiagent biological and social systems [1,2] where the interactions between the individuals are described by the concept of the payoff matrix introduced in traditional game theory [3-5]. In recent decades the methods of statistical physics have been applied comprehensively for the quantitative investigations of phenomena in models where the individuals are distributed on a lattice (or graph) and the interactions are limited to their neighborhoods [6-8]. Previously it was recognized that the ferromagnetic Ising model $[9,10]$ can also be considered as a social (or biological) model [11-14] where the neighboring individuals benefit if they choose the same option (for recent reviews, see Refs. [15-19]). The analogy in the macroscopic behavior is valid if the evolution of strategy distribution is governed by the logit rule [20-22] resembling the Glauber dynamics of the kinetic Ising model [23]. The main advantage of the application of the logit rule is that these systems evolve into the Boltzmann distribution if the pair interactions are defined by potential games for $n$ strategies [19,24-26]. Consequently, at low noises a long-range ordered strategy arrangement is realized that transforms into a random strategy distribution if the noise is increased. These phase transitions are well described by the Ising or Ising-type models.

We have to emphasize, however, that in biologically motivated evolutionary games the realistic dynamical rules are generally based on imitation in agreement with the spirit of Darwinian selection [1]. The application of the imitation dynamical rule results in a significantly different macroscopic behavior because all the homogeneous states become absorbing states [6]. When tuning the interaction parameters the two-strategy systems can exhibit one or two consecutive phase transitions between the two homogeneous states [6,7]. Two transitions occur if the two strategies can coexist in a sufficiently large system. In the latter cases the extinction of the minority strategy follows a universal behavior (belonging to the directed percolation universality class [27-30]) when approaching the critical points [31-33]. At the same time, the numerical investigations [7,19,34] indicated a first-order phase transition for those interactions where the strength of coordination was sufficiently large.

In the models mentioned above the authors used the notation of social dilemmas $[4,35,36]$ when the first and second strategies are called defection and cooperation. The payoffs quantify: punishment for defection $(P=0)$, rewards for mutual cooperation $(R=1)$, temptation to choose defection $(T)$, and sucker's payoff $(S)$. In the latter terminology our investigation is restricted to the region of the stag hunt game $(T<1$ and $S<0)[37,38]$ where both homogeneous pure strategy pairs $[(1,1)$ and $(2,2)]$ are Nash equilibria. Although most of the previous analyses of spatial social dilemmas are focused on the weak prisoner's dilemma $(S=0$ and $T>1)$ [31,33,39], there are some papers considering two-dimensional lattice systems in the whole $T-S$ parameter space for a fixed noise level or using other versions of dynamical rules (for reviews see [6,7]). When varying the payoff parameters, a sudden change between the two homogeneous strategy arrangements was reported by several authors who described that the final absorbing state may depend on the initial strategy distribution [40-42].

Now our investigations will be focused on the motion of interfaces separating the competing homogeneous phases. The visualization of the interface motion indicated anisotropic invasion velocities. Using a simple numerical method we will quantify the average invasion velocities when varying the payoff parameters for two (relevant) orientations. The two-strategy multiagent potential game on a square lattice will 
be investigated by using a convenient parametrization of the interactions. In the present study the interaction is composed of a unit strength coordination game that is extended by selfand cross-dependent components with variable strengths [43]. For the logit dynamical rule this system is equivalent to a two-dimensional Ising model in the presence of a suitable external magnetic field. It is already well known that if the simulations of these systems are started from an artificial initial state in which one of the strategies forms a sufficiently large circular island in the sea of the other strategy, then the growth (or shrinking) of this island only depends on the sign of the external magnetic field. Conversely, if the evolutionary rule is based on stochastic pairwise imitations [31] then our simulations have indicated a surprisingly different behavior. In a range of parameters in which both types of islands are shrinking due to the anisotropic invasion velocity. The latter feature implies the relevance of continuous percolation in the formation of the final absorbing state.

In the next section we describe the model, discuss its general features, and show the unexpected phenomena. The quantitative analysis of the anisotropic invasion velocity is detailed in Sec. III. The relationships between the anisotropic invasion velocity, the phase diagrams including regions of two possible final homogeneous states, and the continuum percolation are discussed in Sec. IV. Finally, we briefly survey the robustness and relevance of these phenomena.

\section{THE MODEL AND ITS GENERAL FEATURES}

We study evolutionary games with equivalent players residing at the sites $x$ of a square lattice with $N=L^{2}$ sites and periodic boundary conditions. Each player residing on site $x$ chooses one of her two options and plays matrix games with her four nearest neighbors (denoted by sites $x+\delta x$ ). If the first and second pure strategies are denoted by two-dimensional Cartesian unit vectors, that is $\mathbf{s}_{x}^{T}=(1,0)$ or $(0,1)$ (superscript $T$ refers to transpose), then the payoff can be described as

$$
u_{x}=\sum_{\delta x} \mathbf{s}_{x} \cdot \mathbf{A} \mathbf{s}_{x+\delta x},
$$

where the summation runs over all the neighboring sites $(x+\delta x)$. In the present work the payoff matrix $\mathbf{A}$ will be defined by two parameters:

$$
\begin{aligned}
\mathbf{A} & =\left(\begin{array}{rr}
1 & -1 \\
-1 & 1
\end{array}\right)+\varepsilon\left(\begin{array}{rr}
1 & 1 \\
-1 & -1
\end{array}\right)+\delta\left(\begin{array}{ll}
1 & -1 \\
1 & -1
\end{array}\right) \\
& =\left(\begin{array}{rr}
1+\varepsilon+\delta & -1+\varepsilon-\delta \\
-1-\varepsilon+\delta & 1-\varepsilon-\delta
\end{array}\right),
\end{aligned}
$$

where the first component refers to a coordination game of unit strength. The second and third components define the self- and cross-dependent payoffs with a strength of $\varepsilon$ and $\delta$, respectively. In this notation the matrix component $A_{i j}$ determines the payoff of player $x$ if she uses the $i$ th strategy while the coplayer chooses the $j$ th one.

It is worth noting that the exchange of strategy labels $(1 \leftrightarrow 2)$ for both players leaves the coordination component unchanged, but reverses the strength of the self- and cross-dependent components $(\delta \rightarrow-\delta$ and $\epsilon \rightarrow-\epsilon$ simultaneously). As a result, the analysis of this system can be constrained to the half-plane (now $\delta>0$ ).
For this parametrization of payoffs the potential matrix [19] obeys the following form:

$$
\mathbf{V}=\left(\begin{array}{cc}
1+2 \varepsilon & -1 \\
-1 & 1-2 \varepsilon
\end{array}\right)
$$

which is independent of $\delta$ (the strength of the cross-dependent component) and the potential of the whole system is given by

$$
U(\mathbf{s})=\frac{1}{2} \sum_{x, \delta x} \mathbf{s}_{x} \cdot \mathbf{V} \mathbf{s}_{x+\delta x},
$$

that summarizes the contributions of pair potentials Eq. (3) for the neighboring pairs only once in each microscopic state $\mathbf{s}=\left\{\mathbf{s}_{x}\right\}$.

If the so-called logit rule [19,24-26] controls the evolution of the strategy distribution then the players can modify their own strategy in a random sequential order exponentially favoring their higher individual payoff with an assumption that the neighboring strategies are fixed. This evolutionary rule drives the system into the Boltzmann distribution, where the probability of the state $\mathbf{s}$ is $p(\mathbf{s})=e^{U(\mathbf{s}) / K} / Z$ and $Z$ is a normalizing factor. Here $K$ quantifies the strength of noise and plays the role of temperature in physical systems.

In the equilibrium at low noises the system prefers the state $\mathbf{s}$ maximizing the value of potential. Accordingly, all the players choose the first (second) strategy if $\varepsilon>0(\varepsilon<0)$ when $K \rightarrow 0$. If $K$ is increased for $\varepsilon=0$, then this system undergoes a critical order-disorder phase transition belonging to the Ising universality class [44]. Indeed, the present evolutionary game is equivalent to the ferromagnetic Ising model with a unit coupling constant, where $\varepsilon$ is the strength of the external magnetic field.

The simplicity of the Ising model is exploited in a wide range of research fields, including the investigation of equilibrium states in magnetic systems, lattice gases, or social systems, as well as the study of processes when the system tends towards the equilibrium state $[10,23,45,46]$. In the magnetic Ising models "spin up" $(\uparrow)$ and "spin down” $(\downarrow)$ states are permitted at the lattice sites. It is well known, for example, that the direction of the motion of the magnetic domain wall (separating the $\uparrow$ and $\downarrow$ regions in the ferromagnetic phase at low values of $K$ ) depends only on the direction of the magnetic field (here the sign of $\varepsilon$ ). As a result, if the magnetic field prefers the formation of $\uparrow$ phase then the islands of states $\downarrow$ shrink and vanish. By contrast, sufficiently large islands of the $\uparrow$ states in the sea of $\downarrow$ states grow and finally a homogeneous state will dominate the system behavior.

In striking contrast to the above scenario, Fig. 1 illustrates a significantly different phenomenon in the presence of the cross-dependent component if stochastic pairwise imitation governs the evolution. Namely, the consecutive snapshots, achieved by Monte Carlo (MC) simulations, clearly show that the circular domains transform into rectangular ones with an orientation dependent on the type of domain. Subsequently, both types of domains shrink and vanish.

First we emphasize that for $\delta>0$ the imitation of the first strategy is beneficial for both the master and its follower because the cross-dependent component provides a higher income for both players. At the same time this advantage is reduced by the self-dependent component if $\varepsilon<0$. The 

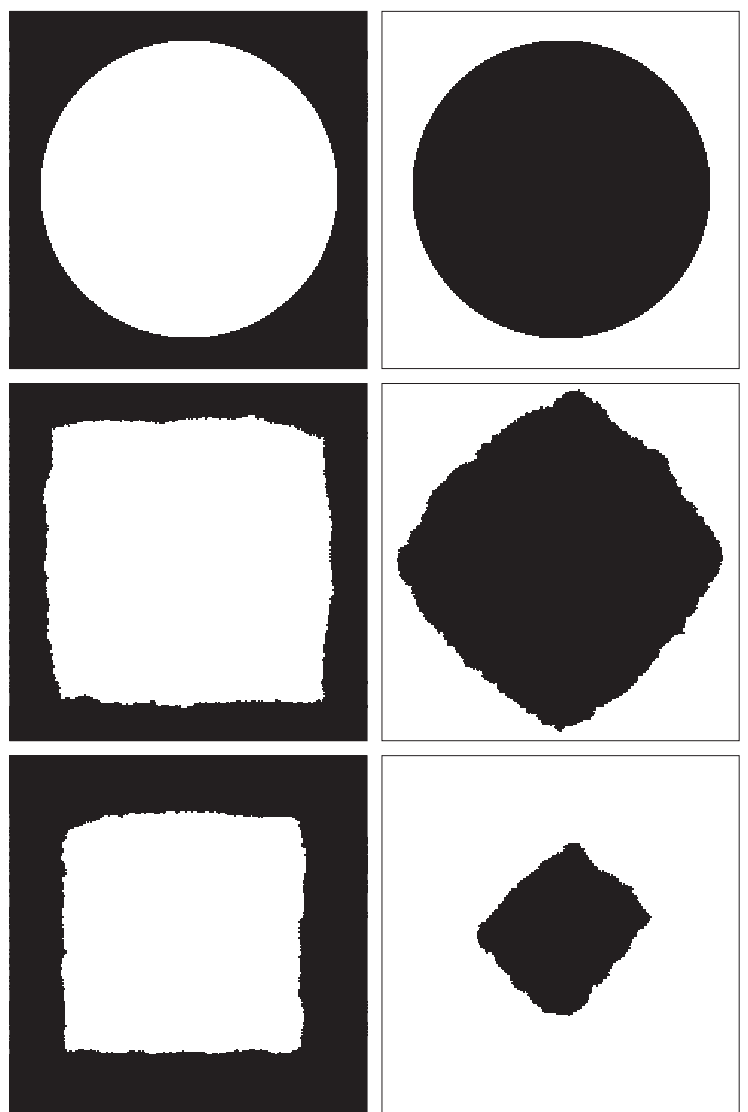

FIG. 1. Evolution of the distribution of strategies 1 (black) and 2 (white) on the square lattice if the MC simulations are started from a state where the minority strategies form a circular domain (with a radius $r=100$ ) as illustrated in the upper two snapshots at $t=0$ MCS. The next two rows of snapshots show the distortion of domains (at $t=500 \mathrm{MCS}$ ) that are shrinking continuously, as it is illustrated in the bottom snapshots at $t=4000$ MCS. These simulations are performed for $\delta=0.5, \varepsilon=-0.03, K=0.3$, and $L=400$.

competition between these opposite effects results in the behavior shown in Fig. 1 when the evolution is governed by repeating the elementary steps described below.

In an elementary step of strategy updates, we first choose two neighboring players (e.g., at sites $x$ and $x+\delta x$ ) and the first player (the follower) adopts the other's (the master's) strategy with a probability [31] depending on the payoff differences as

$$
W\left(\mathbf{s}_{x} \leftarrow \mathbf{s}_{x+\delta x}\right)=\frac{1}{1+e^{\left(u_{x}-u_{x+\delta x}\right) / K}},
$$

that prefers the adoption of the strategy with a higher payoff. In this formula, $K$ quantifies the frequency of mistakes. The above elementary steps are repeated $N$ times in a Monte Carlo step (MCS) during the simulations. For this dynamical rule all homogeneous states are absorbing states, and the other strategy cannot occur inside a homogeneous region. In the limit $K \rightarrow$ $\infty$ this system becomes equivalent to the voter model [47] that exhibits a curious behavior on two-dimensional lattices [48].

The formation of faceted domains was already the subject of intense investigations, because it plays a fundamental role in crystal growth. Most of the related phenomena are well
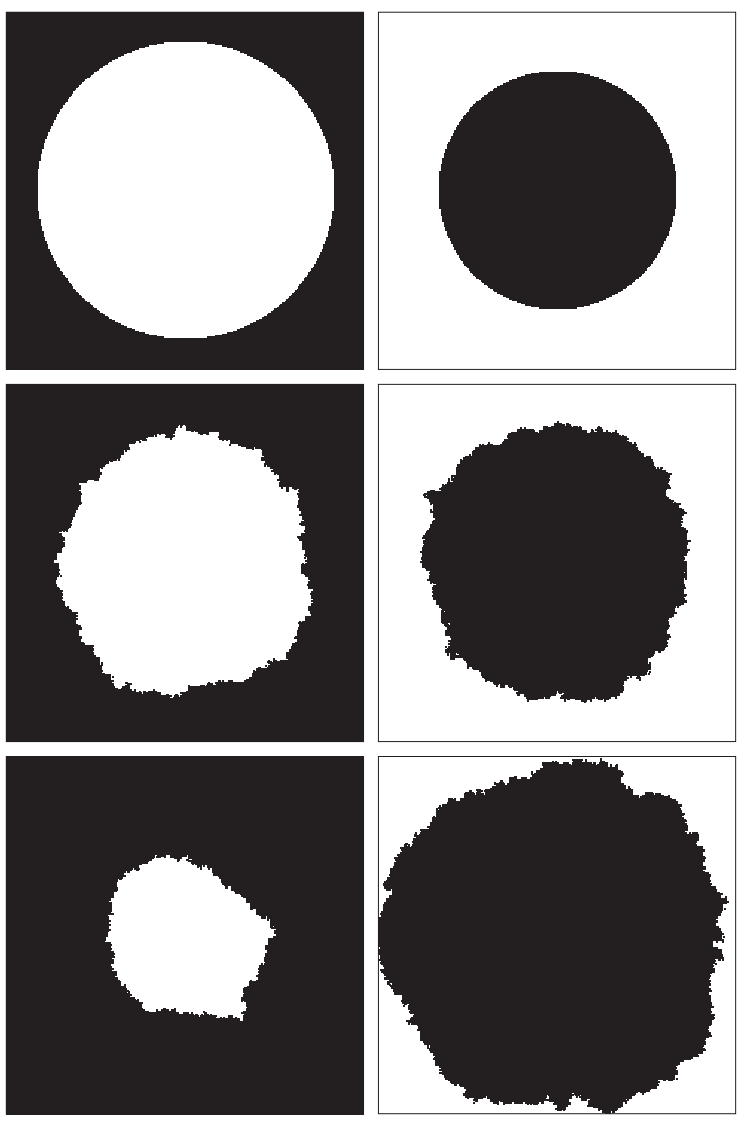

FIG. 2. The sufficiently large and initially circular white (strategy 2) and black (strategy 1) domains exhibit opposite behaviors during the evolutionary process at a high noise level $(K=3)$ for the same payoff parameters we used in the simulations plotted in Fig. 1. The snapshots show the strategy distributions at times $t=0$ (upper), 1000 (middle), and 3000 MCS (lower).

described by suitable Ising models [49,50] and explained by anisotropic interfacial energy, resulting in orientationdependent interfacial velocity in nonequilibrium systems.

At high noises the interfaces become irregular, diminishing thereby the mechanisms yielding anisotropic invasion velocities. The consequences of the high noises are illustrated by two series of snapshots (see Fig. 2) that show how the white circular domain shrinks while the black one grows for the same payoff parameters at a significantly higher noise level.

In light of these results the phenomenon at low noises (see Fig. 1) can be explained by two observations: for the given values of parameters, the white (strategy 2) territories are invaded by the black (strategy 1) ones along horizontal and vertical interfaces. On the other hand, the invasion velocity is opposite along the tilted interfaces with slopes of \pm 1 .

The mentioned features have motivated us to systematically consider the effect of $\delta$ and $\epsilon$ on the average invasion velocities for these two orientations playing a key role in these dynamical processes.

\section{ANISOTROPIC INVASION VELOCITY}

The average invasion velocities are determined by MC simulations for fixed noise levels and system size $(L=1000)$, 

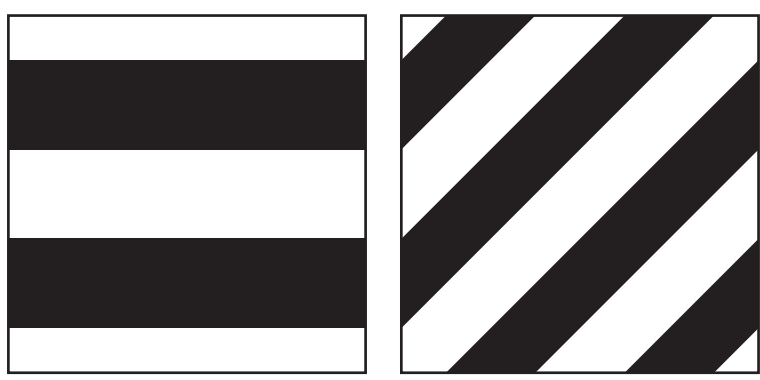

FIG. 3. Horizontal and tilted interfaces in the initial states used for quantifying the interfacial velocities.

with variable payoff parameters $(\delta$ and $\varepsilon$ ). The initial states have been set to have four strip domains (on the torus) with parallel interfaces separating strategies 1 and 2 as it is illustrated in Fig. 3. These states satisfy the periodic boundary conditions and the interfacial energy (on the torus) has a local minimum for both cases.

The numerical analyses are restricted to horizontal and tilted (with a slope of 1) interfaces. During the simulations, we have recorded the portions of strategies, $\rho_{1}(t)$ and $\rho_{2}(t)$ $\left(\rho_{1}(t)+\rho_{2}(t)=1\right)$ and the average velocities are evaluated as the derivatives of $\rho_{2}(t)$ with respect to time $t$ in region where $\rho_{2}(t)$ varies linearly. More quantitatively,

$$
v=\frac{L}{k} \frac{\partial \rho_{2}(t)}{\partial t}
$$

if there are $k$ interfaces in the initial states. To reduce the statistical error this quantity is averaged over a sufficiently long time period and 100 runs. Deviations from the linear decrease and increase of $\rho_{2}(t)$ occurred both in the initial transient period (typically $t<100 \mathrm{MCS}$ ) and when the interfaces collided and annihilated.

As expected, the MC data (see Fig. 4) shows that both types of invasion velocities increase when $\varepsilon$ is decreased. Notice, furthermore, that in the plotted region of $\varepsilon$, the invasion of strategy 2 is always higher along the tilted interfaces, compared to those occurring along the horizontal or vertical ones. The most relevant message of Fig. 4, however, is that we can distinguish two threshold values of $\varepsilon$, namely $\varepsilon_{h}$ and

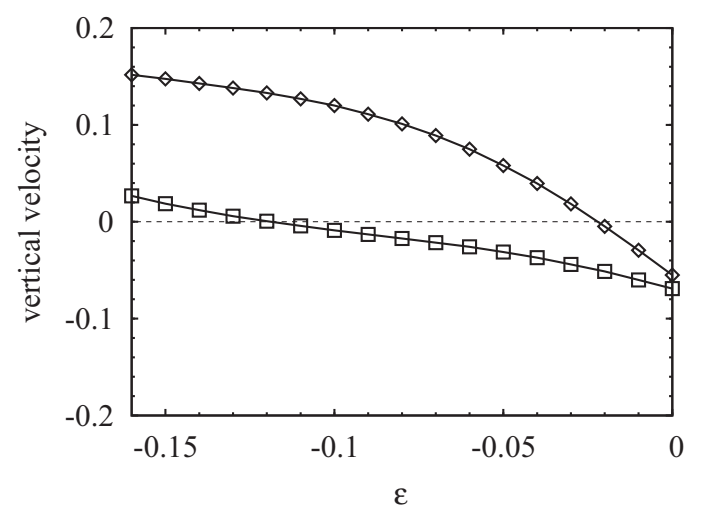

FIG. 4. Average vertical velocity of the horizontal (open boxes) and tilted (open diamonds) interfaces as a function of $\varepsilon$ for $\delta=0.5$ and $K=0.3$. $\varepsilon_{t}$, where the average velocity vanishes along the horizontal and tilted interfaces, respectively. More precisely, strategy 1 invades the territories of strategy 2 , independently of the orientation of interfaces separating them, if $\varepsilon>\varepsilon_{t}$. Conversely, the system will evolve into the homogeneous state of strategy $2\left(\rho_{2}(t)=1\right)$, if $\varepsilon<\varepsilon_{h}$. Quantitatively, $\varepsilon_{t}=-0.0221(3)$ and $\varepsilon_{h}=-0.118(2)$ when $\delta=0.5$ and $K=0.3$.

\section{CONSEQUENCES}

The curious behavior, represented by the snapshots of Fig. 1, can be observed in a narrow range of parameters where the above discussed invasion processes favor opposite strategies. A series of MC simulations have been performed to check the robustness of these unexpected phenomena under different conditions. First, we have repeated the above method to determine the average invasion velocities as a function of $\delta$ and $\varepsilon$ at several noise levels. The results obtained for $K=0.3$ are summarized in a phase diagram (see Fig. 5) where the gray territory denotes the parameters for which the horizontal and tilted interfaces move in opposite directions.

In other words, in the gray territory of the $\delta-\varepsilon$ plane the system can evolve into both homogeneous strategy distributions and the final absorbing state depends on the initial ratio of strategies if $N \rightarrow \infty$. Notice, furthermore, that the sides of symbols indicate the equivalent interface orientations.

The simulations have indicated similar results for $K=0.1$ and 0.6 as plotted in Fig. 6. It is worth emphasizing that the gray territory becomes wider if $K$ is decreased.

During these analyses we faced different technical difficulties increasing the statistical errors and the necessary run times of simulations. For example, at low noise levels the strategy changes occur very rarely along the interfaces, therefore we need long simulations to achieve a sufficiently low relative statistical error. By contrast, for high noise levels the development of interfacial irregularities causes difficulties. In general, the quantification of the average velocities requires the visualization of the pattern evolution in order to exclude the appearance of a coexistence phase along the interfaces. This coexistence phase as well as the two critical phase transitions

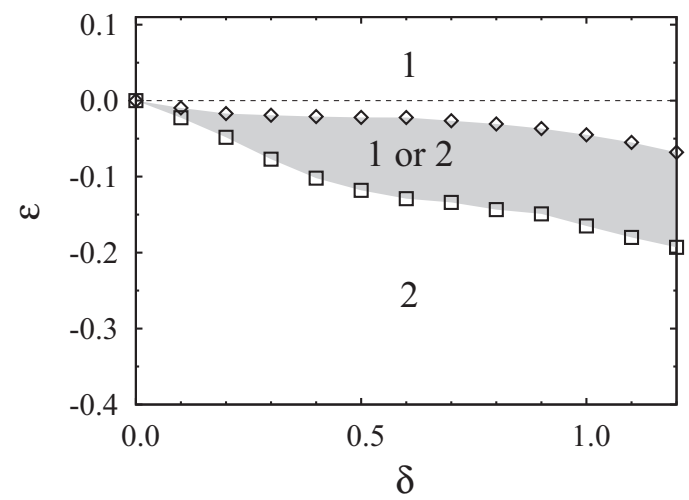

FIG. 5. Boxes and diamonds indicate the values of $\delta$ and $\varepsilon$ where the velocity of the horizontal and tilted interfaces vanish for $K=$ 0.3 . In the gray territory both homogeneous absorbing states can occur when varying the composition of the random initial strategy distribution. 


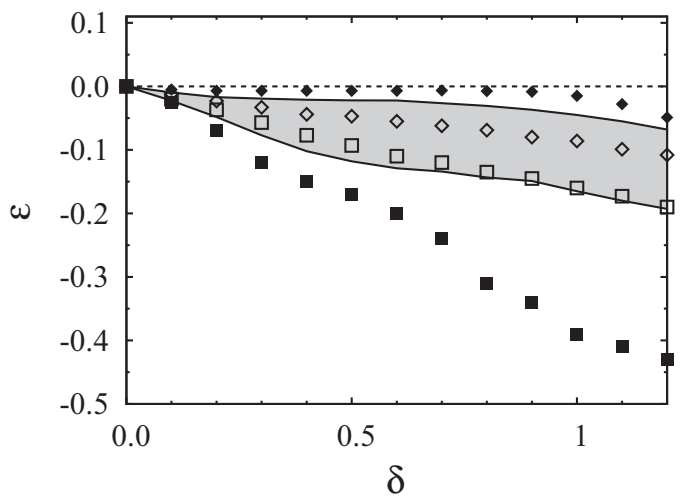

FIG. 6. Boxes and diamonds indicate the threshold values of $\delta$ and $\varepsilon$ where the velocity of the horizontal and tilted interfaces vanish. The filled and open symbols refer to interpolated data obtained for $K=0.1$ and 0.6 , respectively. In order to illustrate the effect of noise, the boundary lines of the gray territory show the same results plotted in Fig. 5 at $K=0.3$.

to one of the absorbing phases were described previously in this system using a different payoff parametrization [7,31-33]. Now the fixed strength of coordination prevents the formation of a coexistence phase on the $\delta-\varepsilon$ plane we studied.

The previous studies have indicated significant variations in the behavior when the square lattice is replaced by other two-dimensional lattices [7,33]. Despite it, similar behaviors are observed when displaying the evolution of circular domains on a square lattice when the players have collected payoffs from games with their nearest and next-nearest neighbors. The striking difference is that the orientations of the faceted shrinking domains are the opposite of those plotted in Fig. 1. These observations have motivated us to check what happens when a triangular lattice (with six neighbors) is used to describe the connectivity structure. In the latter case we could distinguish hexagonal shrinking domains with two types of orientations.

In these cases the invasion processes will eliminate all the islands and the system evolves into the homogeneous state that "first" spans the whole system. The phrase "first" refers to neglecting the transients when the relevance of interfacial invasions is not justified. Such a situation occurs, for example, if the evolution is started from a random initial state, in which case the mean-field approximation gives a more adequate description.

In order to support the above expectation numerically, we ran MC simulations where the system is started from an uncorrelated random initial state with varying the probability of strategy 1, i.e., $\rho_{1}(t=0)$. The typical behaviors for large sizes are illustrated by the MC results of $\rho_{1}(t)$ (see Fig. 7). For large sizes the system evolves into one of the absorbing states dictated by the limit value of $\rho_{1}(t)$ if $t \rightarrow \infty$. The curves plotted in Fig. 7 illustrate that $\lim _{t=\infty}\left[\rho_{1}(t)\right]=1$ (or 0 ) if $\rho_{1}(t)>0.5$ [or $\rho_{1}(t)<0.5$ ] in agreement with the expectations deduced from the results of continuum percolation theory $[51,52]$. To be more specific, in two-dimensional systems the majority phase (it is strategy 1 if $\rho_{1}(t)>1 / 2$ ) spans the system both horizontally and vertically in the thermodynamic limit $(N \rightarrow \infty)[53,54]$. In that case, the minority phase forms

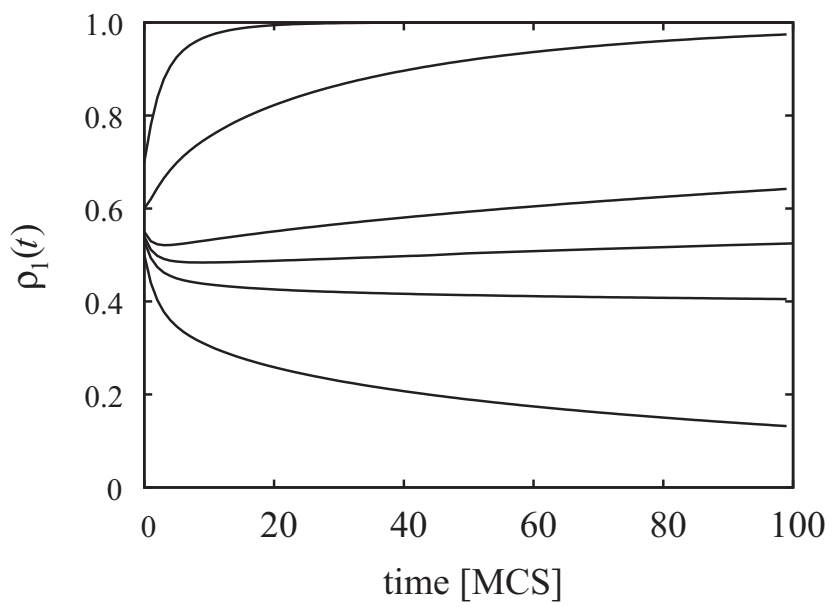

FIG. 7. Time-dependence of the portion of strategy 1 if the simulations are started from $\rho_{1}(t=0)=0.7,0.6,0.55,0.54,0.53$, and 0.5 (from top to bottom) for $\delta=0.5, \varepsilon=-0.03, K=0.3$, and $L=1000$. The plotted MC data are averaged over 100 runs.

shrinking islands, and finally the system evolves into the homogeneous absorbing phase of the majority strategy. The threshold value of $\rho_{1}(t=0)$, separating the limit states if $L \rightarrow \infty$, is estimated as $\rho_{\text {th }} \simeq 0.536(3)$.

For small system sizes, however, the effects of randomness (appearing in the random initial state and also in the consecutive elementary steps) can cause a significant deviation from the limit behavior, particularly in the close vicinity of the threshold values. The latter effect is demonstrated in Fig. 8 where the extinction probabilities as a function of time are plotted for small sizes $L$ in the vicinity of the threshold value, more precisely, when $\rho_{1}(t=0)=0.54$.

These results illustrate that both strategies can become extinct after some time. For a fixed average initial composition the average extinction times depend on the type of strategy and the system size. As the initial value of $\rho_{1}(t=0)$ is slightly above the mentioned threshold value (see the appropriate curve

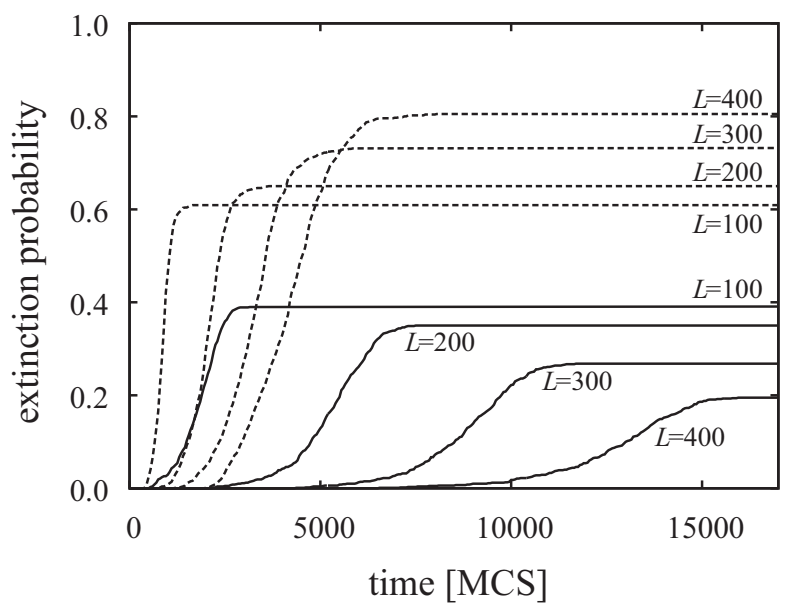

FIG. 8. Extinction probability of strategy 1 (solid lines) and 2 (dashed lines) vs. time for different system sizes (see the labels) for $\rho_{1}(t=0)=0.54, \delta=0.5, \varepsilon=-0.03$, and $K=0.3$. These curves are obtained by averaging over 1000 runs. 
in Fig. 7), the limit value of the extinction probability of strategy 1 tends to zero if $L$ is increased. Notice, furthermore, that the extinction of strategy 2 is faster, in agreement with the snapshots of Fig. 1, indicating a larger invasion velocity along the tilted interfaces.

We emphasize that the mean-field approximation predicts a decrease of $\rho_{1}(t)$ from the symmetric $\left[\rho_{1}(t=0)=\right.$ $\rho_{2}(t=0)=1 / 2$ ] random initial state. This is the reason why $\rho_{\text {th }}>1 / 2$.

\section{SUMMARY}

Evolution of domain structures has been studied in twostrategy evolutionary games with nearest-neighbor interactions on a square lattice when both the interaction and the dynamical rule favor the formation of homogeneous strategy distributions at low noises. For this goal, in the present systematic investigations, the pair interaction is dominated by a coordination game (with a fixed strength) that favors equally the formation of one of the two homogeneous phases. The formation of a homogeneous final state is also supported by the dynamical rule if it is based on imitating one of the more successful neighbors. In the present work, the interaction is extended by self- and cross-dependent components with different strengths denoted by $\varepsilon$ and $\delta$.

For the application of logit rule the pattern evolution in these systems at low noises becomes equivalent to those described by the Ising model in the presence of an external magnetic field. In the corresponding evolutionary games the self-dependent component plays the role of magnetic field; meanwhile, the cross-dependent component does not affect the evolution of domain structure. This scenario is changed drastically when an imitation-type dynamical rule controls the evolutionary process.

For stochastic pairwise imitation the Monte Carlo simulations have indicated that in a certain region of parameters both types of circular domains shrink after evolving into a square-like faceted shape with two different orientations.
This phenomenon can be explained by the opposite invasion velocities along the horizontal (vertical) or tilted (with slopes of \pm 1 ) interfaces. Using strip-like initial states we have determined the average invasion velocities along the relevant interfaces. Additionally, we have determined those regions of the $\delta$ - $\varepsilon$ plane in which the average invasion velocities along the horizontal and tilted interfaces are opposite to each other. In this parameter region both types of strategy islands shrink and vanish sooner or later. Consequently, if a sufficiently large system is started from a random initial state with a prescribed composition then it will evolve into the homogeneous state that percolates first throughout the whole battlefield with periodic boundary conditions. This theoretical expectation as well as the related final size effects are justified by Monte Carlo simulations.

Now it turned out that the competition between the self- and cross-dependent components may result in opposite motions of the interfaces separating the two homogeneous states. The most relevant consequence of this phenomenon is that the system can evolve into both homogeneous absorbing states when tuning the composition of the initial states. We have to emphasize that this feature cannot be explored by using the traditional numerical methods when all simulations are started from symmetric random initial states while the parameters are tuned.

The relevance of the previously discussed phenomena is not limited to the two-strategy spatial evolutionary games since the stochastic strategy imitation can eliminate strategies consecutively as it is already demonstrated in several $n$-strategy systems [34,55-60]. In many cases the formation of the final absorbing state may be determined by the competition of two homogeneous territories, whereas the transient processes are influenced by the additional strategies being present in the initial states.

\section{ACKNOWLEDGMENT}

This work was supported by the Hungarian National Research Fund (Grant No. OTKA TK-120785).
[1] J. Maynard Smith, Evolution and the Theory of Games (Cambridge University Press, Cambridge, UK, 1982).

[2] M. A. Nowak, Evolutionary Dynamics (Harvard University Press, Cambridge, MA, 2006).

[3] J. von Neumann and O. Morgenstern, Theory of Games and Economic Behaviour (Princeton University Press, Princeton, 1944).

[4] K. Sigmund, The Calculus of Selfishness (Princeton University Press, Princeton, NJ, 2010).

[5] W. H. Sandholm, Population Games and Evolutionary Dynamics (MIT University Press, Cambridge, MA, 2010).

[6] G. Szabó and G. Fáth, Phys. Rep. 446, 97 (2007).

[7] C. P. Roca, J. A. Cuesta, and A. Sánchez, Phys. Life Rev. 6, 208 (2009).

[8] B. Allen and M. A. Nowak, EMS Surv. Math. Sci. 1, 113 (2014).
[9] E. Ising, Z. Physik 31, 253 (1925).

[10] C. Domb, in Phase Transitions and Critical Phenomena, Vol. 3, edited by C. Domb and M. S. Green (Academic Press, London, 1974), pp. 357-484.

[11] W. Weidlich, Br. J. Math. Psychol. 24, 251 (1971).

[12] S. Galam, Y. Gefen, and Y. Shapir, Math. J. Sociol. 9, 1 (1982).

[13] A. V. M. Herz, J. Theor. Biol. 169, 65 (1994).

[14] S. M. Krause and S. Bornholdt, Physica A 392, 4048 (2013).

[15] S. N. Dorogovtsev, A. V. Goltsev, and J. F. F. Mendes, Rev. Mod. Phys. 80, 1275 (2008).

[16] C. Castellano, S. Fortunato, and V. Loreto, Rev. Mod. Phys. 81, 591 (2009).

[17] J.-P. Bouchaud, J. Stat. Phys. 151, 567 (2013).

[18] D. Sornette, Rep. Prog. Phys. 77, 062001 (2014).

[19] G. Szabó and I. Borsos, Phys. Rep. 624, 1 (2016). 
[20] G. W. Brown, in Activity Analysis of Production and Allocation, edited by T. C. Koopmans (Wiley, New York, 1951), pp. 373-376.

[21] D. Monderer and L. S. Shapley, J. Econ. Theory 68, 258 (1996).

[22] C. P. Roca, J. A. Cuesta, and A. Sánchez, Eur. Phys. J. B 71, 587 (2009).

[23] R. J. Glauber, J. Math. Phys 4, 294 (1963).

[24] L. E. Blume, Games Econ. Behav. 5, 387 (1993).

[25] L. E. Blume, Games Econ. Behav. 11, 111 (1995).

[26] D. Monderer and L. S. Shapley, Games Econ. Behav. 14, 124 (1996).

[27] T. E. Harris, Ann. Prob. 2, 969 (1974).

[28] P. Grassberger, F. Krause, and T. von der Twer, J. Phys. A 17, L105 (1984).

[29] J. Marro and R. Dickman, Nonequilibrium Phase Transitions in Lattice Models (Cambridge University Press, Cambridge, UK, 1999).

[30] H. Hinrichsen, Adv. Phys. 49, 815 (2000).

[31] G. Szabó and C. Tőke, Phys. Rev. E 58, 69 (1998).

[32] J. R. N. Chiappin and M. J. de Oliveira, Phys. Rev. E 59, 6419 (1999).

[33] G. Szabó, J. Vukov, and A. Szolnoki, Phys. Rev. E 72, 047107 (2005).

[34] G. Szabó and K. Hódsági, Physica A 462, 198 (2016).

[35] M. W. Macy and A. Flache, Proc. Natl. Acad. Sci. USA 99, 7229 (2002).

[36] F. C. Santos, J. M. Pacheco, and T. Lenaerts, Proc. Natl. Acad. Sci. USA 103, 3490 (2006).

[37] M. Starnini, A. Sánchez, J. Poncela, and Y. Moreno, J. Stat. Mech.: Theor. Exp. (2011) P05008.

[38] W. Zhang, Y.-S. Li, C. Cui, and P.-M. Hui, Physica A 443, 161 (2016).

[39] M. A. Nowak and R. M. May, Int. J. Bifurcat. Chaos 3, 35 (1993).
[40] C. Hauert, J. Theor. Biol. 240, 627 (2006).

[41] K. Shigaki, Z. Wang, J. Tanimoto, and E. Fukuda, PLoS ONE 8, e76942 (2013).

[42] J. Tanimoto, Appl. Math. Comput. 263, 171 (2015).

[43] G. Szabó, K. S. Bodó, B. Allen, and M. A. Nowak, Phys. Rev. E 92, 022820 (2015).

[44] H. E. Stanley, Introduction to Phase Transitions and Critical Phenomena (Clarendon Press, Oxford, 1971).

[45] C. Kittel, Introduction to Solid State Physics (John Wiley \& Sons, Chichester, 2004).

[46] A. J. Bray, Adv. Phys. 43, 357 (1994).

[47] T. M. Liggett, Interacting Particle Systems (Springer, New York, 1985).

[48] I. Dornic, H. Chaté, J. Chave, and H. Hinrichsen, Phys. Rev. Lett. 87, 045701 (2001).

[49] C. Rottman and M. Wortis, Phys. Rep. 103, 59 (1984).

[50] V. A. Shneidman, K. A. Jackson, and K. M. Beatty, J. Cryst. Growth 212, 564 (2000).

[51] A. Aharony and D. Stauffer, Introduction to Percolation Theory (Taylor \& Francis, London, 2003).

[52] R. Meester and R. Roy, Continuum Percolation, Cambridge Tracts in Mathematics, Vol. 119 (Cambridge University Press, Cambridge, 1996).

[53] V. Spirin, P. L. Krapivsky, and S. Redner, Phys. Rev. E 63, 036118 (2001).

[54] J. Olejarz, P. L. Krapivsky, and S. Redner, Phys. Rev. Lett. 109, 195702 (2012).

[55] G. Szabó, T. Antal, P. Szabó, and M. Droz, Phys. Rev. E 62, 1095 (2000).

[56] C. Hauert, S. De Monte, J. Hofbauer, and K. Sigmund, Science 296, 1129 (2002).

[57] M. Brede, PLoS ONE 8, e67056 (2013).

[58] P. Cui and Z.-X. Wu, J. Theor. Biol. 361, 111 (2014).

[59] Y. Li, H. Ye, and H. Zhang, Physica A 445, 48 (2016).

[60] Z.-H. Rong, Q. Zhao, Z.-X. W. T. Zhou, and C. K. Tse, Eur. Phys. J. B 89, 166 (2016). 